\title{
Stochastic Optimization Assisted Joint Channel Estimation and Multi-User Detection for OFDM/SDMA
}

\author{
Jiankang Zhang ${ }^{\dagger *}$, Sheng Chen*, Xiaomin $\mathrm{Mu}^{\dagger}$, Lajos Hanzo* \\ $\dagger$ School of Information Engineering, Zhengzhou University, Zhengzhou, China, \\ * School of ECS, University of Southampton, SO17 1BJ, United Kingdom. \\ Tel: +44-23-8059 3125, Fax: +44-23-8059 4508, http://www-mobile.ecs.soton.ac.uk \\ Email: jz09v@ecs.soton.ac.uk, sqc@ecs.soton.ac.uk, iexmmu@zzu.edu.cn, 1h@ecs.soton.ac.uk
}

\begin{abstract}
Stochastic optimization assisted joint Channel Estimation (CE) and Multi-User Detection (MUD) were conceived and compared in the context of multi-user Multiple-Input Multiple-Output (MIMO) aided Orthogonal Frequency-Division Multiplexing/Space Division Multiple Access (OFDM/SDMA) systems. The development of stochastic optimization algorithms, such as Genetic Algorithms (GA), Repeated Weighted Boosting Search (RWBS), Particle Swarm Optimization (PSO) and Differential Evolution (DE) has stimulated wide interests in the signal processing and communication research community. However, the quantitative performance versus complexity comparison of GA, RWBS, PSO and DE techniques applied to joint CE and MUD is a challenging open issue at the time of writing, which has to consider both the continuous-valued $\mathrm{CE}$ optimization problem and the discretevalued MUD optimization problem. In this study we fill this gap in the open literature. Our simulation results demonstrated that stochastic optimization assisted joint CE and MUD is capable of approaching both the Cramer-Rao Lower Bound (CRLB) and the Bit Error Ratio (BER) performance of the optimal ML-MUD, respectively, despite the fact that its computational complexity is only a fraction of the optimal ML complexity.
\end{abstract}

Index Terms-Orthogonal frequency division multiplexing (OFDM), space division multiple access (SDMA), channel estimation, multiuser detection, stochastic optimization algorithm.

\section{INTRODUCTION}

In recent years, multiple antennas have been employed both at the transmitter and/or the receiver for achieving various design goals [1], such as maximizing the attainable multiplexing gain, maximizing the number of users supported or maximizing the achievable diversity gain. As one of the most wide-spread multiple antenna aided systems, Orthogonal Frequency-Division Multiplexing/Spatial Division Multiple Access (OFDM/SDMA) [2] exploits the advantages of both OFDM and SDMA, which increase the attainable spectral efficiency by sharing the same bandwidth and time slots by several users roaming in different geographical locations, which are differentiated by their unique, user-specific 'spatial signature', i.e. by their Channel Impulse Responses (CIRs).

More specifically, the transmitted signals of $U$ simultaneous singleantenna aided UpLink (UL) Mobile Stations (MSs) are received by an array of antennas at the Base Station (BS), where MultiUser Detection (MUD) techniques are invoked for separating the signals of the different MSs with the aid of their unique, user-specific 'spatial signature', i.e. CIRs. Naturally, for near-single-user MUD the CIRs have to be accurately estimated [1,3]. Intensive research efforts have been devoted to developing efficient approaches for Channel Estimation (CE) in multi-user OFDM/SDMA systems [1, 4-6]. In order to achieve a near-optimal performance, joint CE and signal detection schemes have recently received significant research attention [7-9]. The optimal solutions of CE and/or MUD, namely Maximum-likelihood (ML) CE and ML-MUD, are naturally desired. However, we have to settle for suboptimal solutions due to the excessive computational complexity of the optimal ML solutions,

Acknowledgments: The financial support of the EPSRC under the auspices of the China-UK Science Bridge as well as of the RC-UK under the India-UK Advanced Technology centre initiative is gratefully acknowledged. especially for a high number of users/antennas relying on Quadrature Amplitude Modulation (QAM).

Fortunately, stochastic optimization algorithms are capable of finding the globally optimal solution with a high probability at a fraction of the optimal ML MUD's complexity, even for problems associated with a non-smooth Cost Function (CF) exhibiting multiple local optima. The most popular algorithms ${ }^{1}$ include Genetic Algorithms (GA) [12], Repeated Weighted Boosting Search (RWBS) [13], Particle Swarm Optimization (PSO) [14] and Differential Evolution (DE) [15]. More specifically, significant advances have been made in the development of these stochastic optimization algorithms, including single-user joint channel and data estimation [13,16], CE and/or MUD in the multi-user Code Division Multiple Access (CDMA) UpLink (UL) [17-20], in the SDMA aided OFDM UL [1,7,9], in MUD assisted Space-Time Block Coding (STBC) [21, 22], in CE for Multiple Input Multiple Output (MIMO) systems [23], in the MultiUser Transmission (MUT) aided DownLink (DL) [24, 25], in channel allocation $[26,27]$ as well as in a diverse range of other applications.

In general, the optimization problems in communications may be classified as: continuous and discrete optimization problems. For example, the CIRs to be estimated are continuous-valued, while the transmitted signals are discrete. To the best of our knowledge, no performance versus complexity comparisons of GA, RWBS, PSO and DE techniques applied to joint CE and MUD have been presented in the open literature.

Against this background, our new contribution is that we provide a performance versus complexity comparison of stochastic optimization algorithms in the context of joint CE and MUD in ODFM/SDMA systems. More specifically, continuous stochastic optimization algorithms will be employed for $C E$, relying on Continuous GA assisted $C E$ (CGA-CE), Continuous RWBS assisted CE (CRWBS-CE), Continuous PSO assisted CE (CPSO-CE) and Continuous DE assisted CE (CDE$C E)$. By contrast, the discrete binary version of the corresponding stochastic optimization algorithms will be employed for MUD, invoking Discrete Binary GA assisted MUD (DBGA-MUD), Discrete Binary RWBS assisted MUD (DBRWBS-MUD), Discrete Binary PSO assisted MUD (DBPSO-MUD) and Discrete Binary DE assisted $M L$ $M U D(D B D E-M U D)$.

The rest of this paper is organized as follows. The system model of the multi-user OFDM/SDMA UL is described in Section II. Section III is devoted to the optimization problems of joint CE and MUD in the OFDM/SDMA systems considered. In Section IV, we will briefly characterize the proposed stochastic optimization algorithms. Our simulation results and discussions are presented in Section V, while our conclusions are offered in Section VI.

\section{System Model}

In the OFDM/SDMA UL systems, $U$ simultaneous users are equipped with a single transmission antenna, while the BS employs

\footnotetext{
${ }^{1}$ There are numerous other stochastic optimization algorithms, such as the Ant Colony [10] and other evolutionary algorithms [11], but given our limited space, we concentrate on the above four algorithms in this paper.
} 


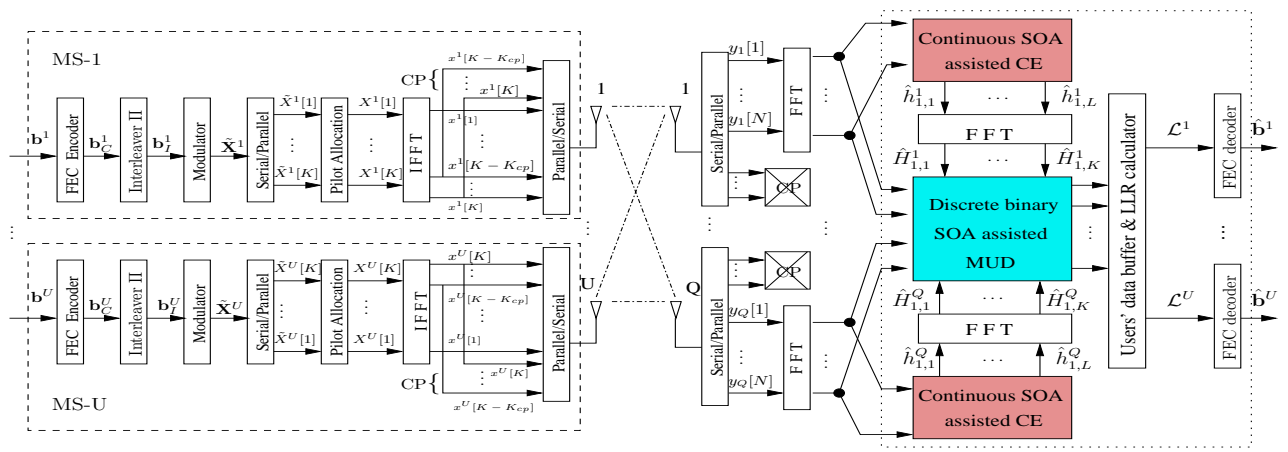

Fig. 1. Uplink system model for Multi-user MIMO OFDM/SDMA. The abbreviation of 'SOA' represents 'stochastic optimization algorithm' in this figure.

an array of $Q$ antennas. All users simultaneously transmit their independent data streams, denoted by $\mathbf{b}^{u}, u=1,2, \cdots, U$. The information bits $\mathbf{b}^{u}$ are first encoded by a user-specific independent Forward Error Correction (FEC) encoder, as seen in Fig. 1. The bit stream output by the FEC encoder is grouped and modulated to a stream of $M$-QAM symbols. The modulated data $\tilde{\mathbf{X}}^{u}$ of Fig. 1 are then Serial to Parallel (S/P) converted and the pilot symbols are embedded into the Frequency Domain (FD) representation of the OFDM symbol. These FD pilot symbols and their specific allocation are known at the receiver and hence can be exploited for CE. The parallel modulated data are further fed to a classic $K$-point Inverse Fast Fourier Transform (IFFT) based modulator in order to generate the Time-Domain (TD) modulated signal. After concatenating the Cyclic-Prefix (CP) of $K_{c p}$ samples, the resultant sequence is transmitted through the MIMO channel and contaminated by the receiver's Additive White Gaussian Noise (AWGN).

At the BS, as illustrated at the right-hand side of Fig. 1, the received signals $\mathbf{y}_{q}$ of antenna $q, q=1,2, \cdots, Q$, are Parallel to Serial $(\mathrm{P} / \mathrm{S})$ converted and then the CPs are discarded from every OFDM symbol. The resultant signals are fed into the corresponding Fast Fourier Transform (FFT)-based receiver of Fig. 1. Let $Y_{q}[s, k]$ denote the signal received by the $q$-th receiver antenna element in the $k$-th subcarrier of the $s$-th OFDM symbol, which is given as the superposition of the different users' channel-impaired received signal contributions plus the AWGN, expressed as:

$$
Y_{q}[s, k]=\sum_{u=1}^{U} H_{q}^{u}[s, k] X^{u}[s, k]+W_{q}[s, k],
$$

where $H_{q}^{u}[s, k]$ denotes the FD-CHannel Transfer Function (FDCHTF) of the link between the $u$-th user and the $q$-th receiver antenna in the $k$-th subcarrier of the $s$-th OFDM symbol. The received signals are then forwarded to the stochastic optimization assisted joint CE and MUD. Then the Log-Likelihood Ratios (LLRs) are generated and forwarded to the $U$ independent FEC decoders.

\section{Optimization Problems of Joint CE AND MUD}

In the context of the joint $\mathrm{CE}$ and MUD of the OFDM/SDMA systems, the optimization problems can be formulated based on the Log-Likelihood Function (LLF) conditioned both on the matrix $\mathbf{h}[s]$ containing the CIR coefficients and on the users' transmitted data $\mathbf{X}[s]$, which is given by

$$
J(\mathbf{h}[s], \mathbf{X}[s])=\sum_{q=1}^{Q}\left\|\mathbf{Y}_{q}[s]-\mathbf{X}^{T}[s] \mathbf{F} \mathbf{h}_{q}[s]\right\|^{2},
$$

where the received data $\mathbf{Y}_{q}[s] \in \mathbb{C}^{K \times 1}$ is a column vector hosting the subcarrier-related variables $Y_{q}[s, k]$. The transmitted data matrix $\mathbf{X}[s] \in \mathbb{C}^{U K \times K}$, the block-diagonal matrix $\mathbf{F} \in \mathbb{C}^{U K \times U L}$ and the CIRs $\mathbf{h}_{q}[s] \in \mathbb{C}^{U L \times 1}$ are given by

$$
\begin{aligned}
\mathbf{X}[s] & =\left[\mathbf{X}^{1}[s], \mathbf{X}^{2}[s], \cdots, \mathbf{X}^{U}[s]\right]^{T}, \\
\mathbf{F} & =\operatorname{diag}\left\{\mathbf{F}^{1}, \mathbf{F}^{2}, \cdots, \mathbf{F}^{U}\right\}, \\
\mathbf{h}_{q}[s] & =\left[\mathbf{h}_{q}^{1 T}[s], \mathbf{h}_{q}^{2 T}[s], \cdots, \mathbf{h}_{q}^{U T}[s]\right]^{T} .
\end{aligned}
$$

Furthermore, $\mathbf{X}^{u}[s] \in \mathbb{C}^{K \times K}$ is a diagonal matrix with elements given by $X^{u}[s, k]$, where $k=1,2, \cdots, K, \mathbf{F}^{u} \in \mathbb{C}^{K \times L}$ denotes the FFT matrix [28], while $\mathbf{h}_{q}^{u}[s] \in \mathbb{C}^{L \times 1}$ represents the CIR vector containing the $L$ significant CIR coefficients.

The joint ML optimization defined in Equation (2) is computationally prohibitive, especially for high-dimensional multi-user systems employing many antennas and high-order $M$-QAM. The complexity of this optimization process may be reduced to a tractable level by invoking an iterative search loop, which explores first the entire set of possible CIRs $\mathbf{h}[s]$ commencing from the initial estimate generated with the aid of pilot symbols and then the set of all the possible transmitted data symbols $\mathbf{X}[s]$, while relying on the estimated CIRs, which may be formulated as:

$$
(\hat{\mathbf{h}}[s], \hat{\mathbf{X}}[s])=\arg \min _{\mathbf{X}[s]}\left[\min _{\mathbf{h}[s]} J(\mathbf{h}[s], \mathbf{X}[s])\right] .
$$

\section{A. Channel Estimation}

When the channel statistics are unknown and the CIRs are treated as deterministic parameters, the ML CF may be minimized, assuming that the CIRs were found with the aid of pilot symbols. Furthermore, the CIRs of $\hat{\mathbf{h}}_{q}[s]$ are valid for the signals $\mathbf{Y}_{q}[s]$ recorded at the $q$-th receiver antenna. Hence, the CF of the channel estimation is equivalent to the minimization of

$$
\begin{aligned}
\hat{\mathbf{h}}_{q}[s] & =\arg \min _{\mathbf{h}_{q}[s]} J\left(\mathbf{h}_{q}[s]\right) \\
& =\arg \min _{\mathbf{h}_{q}[s]}\left\|\mathbf{Y}_{q}[s]-\mathbf{X}^{T}[s] \mathbf{F} \mathbf{h}_{q}[s]\right\|^{2} .
\end{aligned}
$$

\section{B. The $M L-M U D$}

As a benefit of the CP, the OFDM/SDMA symbols do not overlap and hence SDMA MUD processing can be applied on a per-carrier basis $[1,2]$. Hence, the task of the MUD is to recover the transmitted signals $\mathbf{X}[s, k] \in \mathbb{C}^{U \times 1}$ of the $U$ users from the received signals formulated in Equation (1). Each element of $\mathbf{X}[s, k]$, say $X^{u}[s, k]$, belongs to a finite alphabet $\mathcal{S}$ of size $|\mathcal{S}|=M$. Hence there are $M^{U}$ possible candidate solutions $\hat{\mathbf{X}}[s, k]$. The optimal MLMUD exhaustively searches the full space of $\mathcal{S}^{U}$ to find a solution minimizing $\|\mathbf{Y}[s, k]-\mathbf{H}[s, k] \mathbf{X}[s, k]\|^{2}$, which is equivalent to

$$
\begin{aligned}
& \hat{\mathbf{X}}_{M L-M U D}[s, k]=\arg \min _{\mathbf{X}[s, k] \in \mathcal{S}^{U}} J(\mathbf{X}[s, k]) \\
& =\arg \min _{\mathbf{X}[s, k] \in \mathcal{S}^{U}}\|\mathbf{Y}[s, k]-\mathbf{H}[s, k] \mathbf{X}[s, k]\|^{2} .
\end{aligned}
$$

The above problem may also be viewed as a finite-alphabetconstrained least-squares (LS) problem [29], which is known to be a Nondeterministic Polynomial-time (NP)-hard problem.

\section{Stochastic Optimization Assisted CE And MUD}

Let us now employ the above-mentioned continuous stochastic optimization algorithms for assisting CE, by invoking the CDE-CE, CRWBS-CE, CPSO-CE as well as CGA-CE techniques, which will be combined with discrete binary stochastic optimization algorithms for assisting MUD, namely with the DBDE-MUD, DBRWBS-MUD, DBPSO-MUD as well as DBGA-MUD. Given our limited space, 
we will only briefly introduce the basic philosophy of the stochastic optimization algorithms considered. Readers who are unfamiliar with these algorithms might like to consult their detailed illustrations in the references provided.

\section{A. Basic Philosophy of the Considered Stochastic Optimization Al- gorithms}

The GA philosophy was developed by Holland [12], which constitutes an optimization and search technique inspired by the principles of genetics and natural selection. A salient feature of GAs is that they are capable of searching through the candidate solution space by gradually evolving a pseudo-random initial population through the affordable number of generations by appropriately combining the individual candidate solutions upon exploiting the survival of the fittest individuals by selection, by crossover, by mutation, etc.

Chen et al. developed the RWBS [13] motivated by their experience with GAs and Adaptive Simulated Annealing (ASA). The basic philosophy of the RWBS algorithm is that by commencing from an initially randomly populated search-pool of the potential solutions, RWBS strives for replacing the 'lowest-quality' solutions of the population with the "best" potential solutions generated by natureinspired combinations/mutations of the candidate solutions in the pool, until the process converges. The process is constituted by an amalgam of the so-called mutation, evaluation, normalization, weighting and construction steps [9].

The PSO algorithm [14] is also a population based stochastic optimization technique inspired by mimicking the social behavior of organisms such as fish schooling and bird flocking. The individuals are known as particles in PSO terminology. PSO has no evolutionary operators, such as the crossover and mutation. Instead, it refines its search by attracting the independent variables referred to as particles in PSO parlance to specific positions associated with high fitness, i.e. with near-ML solutions. This is carried out by adjusting the particles' flight trajectory according to both their own and their companions' past trajectory.

The DE algorithm [15] is a relatively new member in the family of Evolutionary Algorithms (EAs), which has its own distinctive features. Specifically, it mutates the candidate-solution vectors by adding weighted, random difference-vectors to them, which assist them in promptly approaching the globally optimal solution.

\section{B. Computational Complexity}

A low-complexity search-termination criterion is constituted by the number of CF Evaluations (CF-Evals.), which may be readily used for estimating the computational complexity imposed. For a given population size $P_{s}$ terminated after $G$ generations, the number of CF-Evals. employed by the stochastic optimization algorithms for finding the solution representing the detected users' signals (or estimated CIRs) is equal to $\left(P_{s} \times G\right)$ for the $\mathrm{DE}$, for the $\mathrm{GA}$ as well as for the PSO, while it is $\left[\left(P_{s}+2 T_{b s}\right) \times G\right]$ for the RWBS, where $T_{b s}$ is the number of boosting search steps at each generation [9]. By contrast, the number of CF-Evals. of the optimum ML-MUD using exhaustive search is equivalent to $M^{U}$ for $U$ MSs supported in the OFDM/SDMA UL in conjunction with $M$-QAM. Hence, in contrast to the ML solution, the complexity of the stochastic optimization assisted MUDs is not directly dependent on the number of bits/symbols in $M$-QAM and on the number of user supported, which indicates that they are particularly beneficial in large-dimensional multi-user systems employing high-order $M$ QAM.

\section{Simulation Results}

In this section, our simulation results are presented in order to characterize the stochastic optimization assisted joint CE and MUD
TABLE I

THE ALGORITHMIC PARAMETERS FOR THE STOCHASTIC OPTIMIZATION ALGORITHMS ASSISTED CE AND MUD

\begin{tabular}{|c|l|l|}
\hline \hline Schemes & Parameters & Values \\
\hline \multirow{3}{*}{ CDE-CE } & Population size $P_{s}$ & 100 \\
& Greedy factor $p$ & 0.1 \\
& Adaptive update factor $c$ & 0.1 \\
\hline \multirow{3}{*}{ CRWBS-CE } & Population size $P_{s}$ & 100 \\
& Mutation parameter $\gamma$ & 0.001 \\
& Boosting search times $T_{b s}$ & 40 \\
\hline \multirow{3}{*}{ CPSO-CE } & Population size $P_{s}$ & 100 \\
& Cognition learning factor & 2 \\
& Social learning factor & 2 \\
\hline \multirow{3}{*}{ CGA-CE } & Population size $P_{s}$ & 100 \\
& Selection ratio $r_{s}$ & 0.5 \\
& Mutation probability $M_{b}$ & 0.2 \\
\hline \hline \multirow{2}{*}{ DBDE-ML-MUD } & Population size $P_{s}$ & 100 \\
& Greedy factor $p$ & 0.7 \\
& Adaptive update factor $c$ & 0.8 \\
\hline \multirow{2}{*}{ DBRWBS-ML-MUD } & Population size $P_{s}$ & 100 \\
& Mutation probability $M_{b}$ & 0.5 \\
& Boosting search times $T_{b s}$ & 40 \\
\hline \multirow{2}{*}{ DBPSO-ML-MUD } & Population size $P_{s}$ & 100 \\
& Cognition learning factor & 0.1 \\
& Social learning factor & 0.3 \\
\hline \multirow{2}{*}{ DBGA-ML-MUD } & Population size $P_{s}$ & 100 \\
& Selection ratio $r_{s}$ & 0.5 \\
& Mutation probability $M_{b}$ & 0.15 \\
\hline \hline
\end{tabular}

considered in the context of multi-user OFDM/SDMA systems. It was assumed that the UL multi-user OFDM/SDMA system equipped with $Q=4$ antennas at the BS supported $U=4$ MSs simultaneously transmitting their data in the UL to the BS. Moreover, a half-rate Recursive Systematic Convolutional Code (RSC) having the generator polynomial $(7,5)$ and the constraint length $C L=3$ is employed as FEC. For the sake of a fair comparison, a large number of Monte-Carlo simulations have been run for finding the appropriate algorithmic parameters for the stochastic optimization assisted joint CE and MUD considered. A summary of the default values for the various parameters used in our simulations is provided in Table I. The values of these parameters were set to the default values throughout the paper, unless specified differently in the figures or in the figure captions.

Important observations may be inferred from Fig. 2 and Fig. 4 about the number of CF-Evals. and the reliability of the different stochastic optimization assisted joint $\mathrm{CE}$ and MUD, where the reliability was quantified in terms of the probability of successfully identifying the ML solution. In order to have a unified criterion of 'successful identification', we assume encountering a perfect channel, i.e. that there is no channel-induced impairments are imposed ${ }^{2}$. Then the 'successful identification' is defined as the condition of having a CF value, which is within a margin of $J\left(\hat{\mathbf{h}}_{q}[s]\right)<10^{-4}$ and $J(\hat{\mathbf{X}}[s, k])<10^{-4}$ for the stochastic optimization assisted CE and MUD within the affordable computational complexity. Quantitatively, we fixed the number of CF-Evals. to $1.0 \times 10^{5}$ and $5.0 \times 10^{4}$ for the $\mathrm{CE}$ and MUD procedures, respectively. Otherwise, an 'identification failure' is declared. We can see from Fig. 2 that the CDE-CE outperforms all the other three schemes and always arrives at $J\left(\hat{\mathbf{h}}_{q}[s]\right)<$ $10^{-4}$ within the maximum affordable computational complexity of CF-Evals. $=1.0 \times 10^{5}$. By contrast, the CGA-CE has a higher computational complexity and yet, it exhibits a higher failure ratio in estimating the CIRs within the pre-defined maximum affordable

\footnotetext{
${ }^{2}$ Note that similar trends would also be observed in the presence of statistic channels. However, it would be challenging to define the 'successful identification' for the CE and MUD, since they both exhibit a lower-bound, which is given by the CRLB and by the BER of the optimal ML-MUD for the stochastic optimization assisted CE and the stochastic optimization assisted MUD, respectively. The achievable minima of the CFs depend also on the specific data conveyed by the OFDM symbols even at the same $E_{b} / N_{0}$, owning to the random nature of the AWGN.
} 


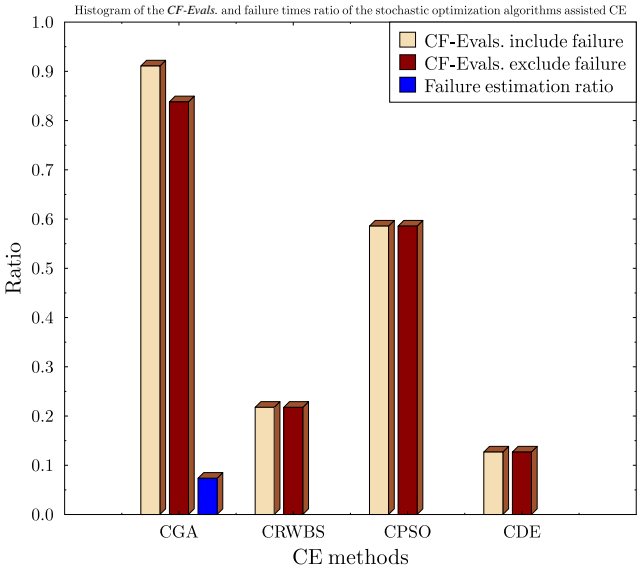

Fig. 2. Histogram of the CF-Evals. and failure times ratio of different stochastic optimization algorithm assisted CEs. The quantity 'Ratio' on the $y$-axis, represents the proportion of experiments imposed a certain CF-Evals. of the pre-defined maximum affordable complexity $\left(P_{s} \times G=100 \times 1000\right)$ and failure times of the total times of independent experiments, respectively.

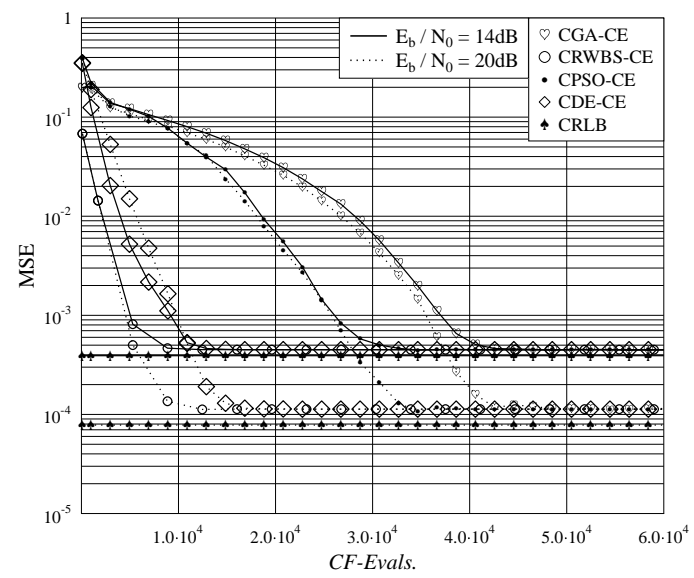

Fig. 3. MSE performance versus the number of CF-Evals. for the different stochastic optimization assisted CEs, which characterizes out the convergence speed of these schemes.

computational complexity of CF-Evals. $=1.0 \times 10^{5}$. Observe in Fig. 4 that DBGA-MUD is the 'winning' MUD candidate, requiring the lowest number of CF-Evals., which is about $3.14 \%$ of that of the optimal ML-MUD. Additionally, the DBGA-MUD detected the users' signals correctly with the highest probability within the predefined maximum affordable computational complexity of CF-Evals. $=5.0 \times 10^{4}$.

In Fig. 3 and Fig. 5 we characterize the MSE and BER versus the number of $C F$-Evals. for the stochastic optimization assisted joint $\mathrm{CE}$ and MUDs at $E_{b} / N_{0}=14 \mathrm{~dB}$ and $E_{b} / N_{0}=20 \mathrm{~dB}$. The achievable lower-bounds of the MSE for the continuous stochastic optimization assisted CE as well as for the BER of the discrete binary stochastic optimization assisted MUD are given by the CRLB and by the BER of the optimal ML-MUD, respectively. Similar trends are also observed at other values of $E_{b} / N_{0}$. It can be seen from Fig. 3 that the CRWBS-CE and CDE-CE significantly outperform the CPSO-CE and CGA-CE techniques, since the former two converge at a faster rate, requiring about CF-Evals. $\approx 14000$. Observe in Fig. 5 that the DBDE-MUD initially converges slowly. However, its convergence is the fastest after $C F$-Evals. $\approx 2500$. Furthermore, the DBDE-MUD, DBRWBS-MUD and DBGA-MUD regimes correctly detect the users' transmitted signals at $E_{b} / N_{0}=20 \mathrm{~dB}$. By contrast, the DBPSO-MUD exhibits error-floors at $B E R \approx 8.72 \times 10^{-3}$ and $B E R \approx 2.93 \times 10^{-4}$ for $E_{b} / N_{0}=14 d B$ and $E_{b} / N_{0}=20 d B$, respectively, since the DBPSO-MUD has a higher probability of

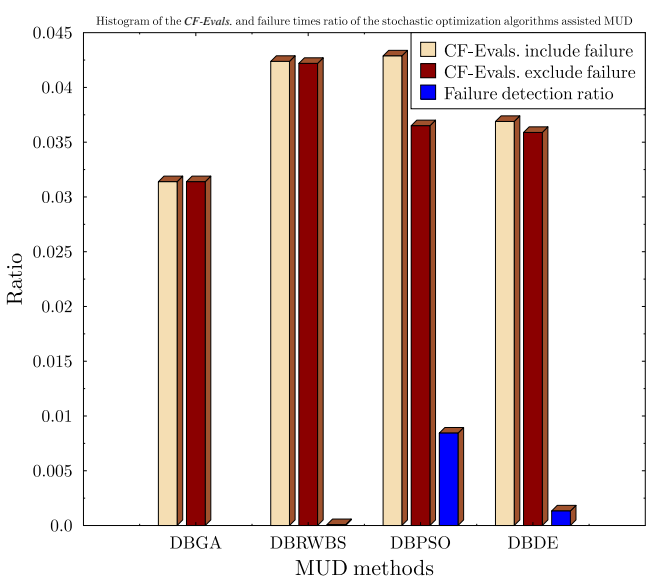

Fig. 4. Histogram of the CF-Evals. and failure times ratio of different stochastic optimization algorithm assisted MUDs. The quantity 'Ratio' on the $y$-axis, represents the proportion of experiments imposed a certain $C F$ Evals. of the optimal ML-MUD $\left(M^{U}=16^{4}\right)$ and failure times of the total times of independent experiments, respectively.

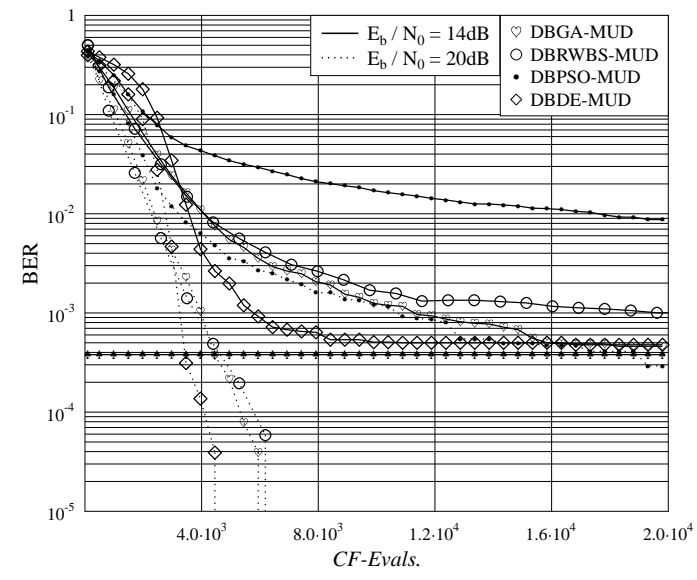

Fig. 5. BER performance versus the number of CF-Evals. for the different stochastic optimization assisted MUDs, which characterizes the convergence speed of these schemes. Note that for $E_{b} / N_{0}=20 d B$, the optimal ML-MUD attains an infinitesimally low BER, which is not shown in this figure.

failing to find the ML solution, as seen from Fig. 4.

In order to provide an overall impression of the system's performance, we portray the attainable MSE and BER performance in Fig. 6 and Fig. 7, respectively. We evaluate the continuous-valued stochastic optimization assisted $\mathrm{CE}$ at the affordable computational complexity of both ${ }^{3} C F$-Evals. $=20000$ and CF-Evals. $=50000$. We can see from Fig. 6 that the CDE-CE and CRWBS-CE techniques exhibit a similar performance at these two fixed $C F$-Evals. By contrast, at the affordable computational complexity of CF-Evals. $=20000$, the achievable performance of the CPSO-CE and CGA-CE schemes does not improve upon increasing the $E_{b} / N_{0}$ value, in fact, it even degrades at some values of $E_{b} / N_{0}$. Observe in Fig. 7 that as expected, the performance of the discrete-valued binary stochastic optimization assisted MUD relying on CF-Evals. $=10000$ is better than at $C F$ Evals. $=5000$, where the DBDE-MUD is capable of approaching the optimal ML-MUD's performance.

${ }^{3}$ Here we evaluate the continuous-valued stochastic optimization assisted CE's performance at a computational complexity of CF-Evals. $=20000$ and $C F$-Evals. $=50000$. By contrast, we characterize the discrete-valued binary stochastic optimization assisted MUD's performance at a computational complexity of CF-Evals. $=5000$ and CF-Evals. $=10000$. The reason for this is that the discrete binary stochastic optimization assisted MUD requires less CF-Evals. to detect the users' transmitted signals, as seen from Fig. 3 and Fig. 5. 


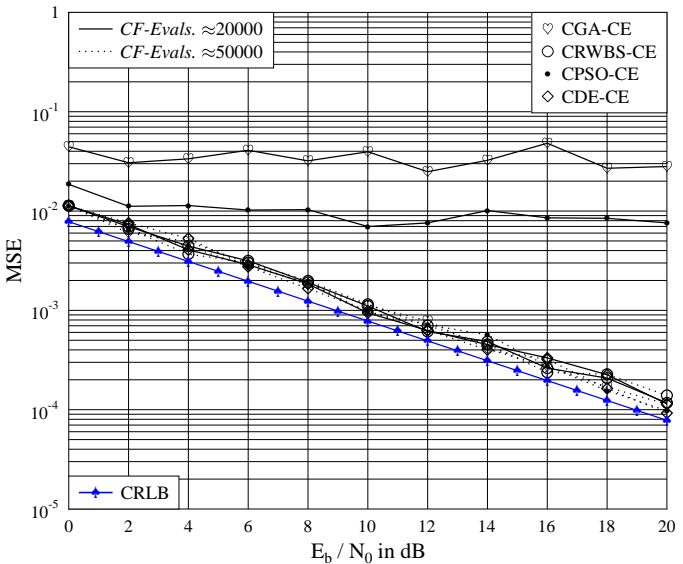

Fig. 6. MSE performance of different continuous-valued stochastic optimization assisted CEs using the parameters of Table I. The CRLB characterizes the best achievable performance of an unbiased estimator, hence we include it as a lower bound benchmark. The more data is used by the CE, the lower the CRLB achieved by the CE [30]. Here we assume that only the original pilots are available for $\mathrm{CE}$.

\section{CONCLUSIONS}

In this paper, we compared the achievable performance versus computational complexity of the DE, RWBS, PSO and GA in the context of joint CE and MUD of ODFM/SDMA systems. The CGACE imposed a higher computational complexity and exhibited a slower convergence. By contrast, the CDE-CE performed best, despite imposing the lowest computational complexity and achieving the highest convergence speed. Furthermore, the DBGA-MUD imposed a lower computational complexity than the other three schemes and exhibited a higher convergence speed. In conclusion, the best MSE and BER performance was attained by the DE technique in the context of the system considered.

\section{REFERENCES}

[1] M. Jiang and L. Hanzo, "Multiuser MIMO-OFDM for next-generation wireless systems," Proceedings of the IEEE, vol. 95, no. 7, pp. 14301469, 2007.

[2] P. Vandenameele, L. Van Der Perre, M. G. E. Engels, B. Gyselinckx, and H. J. De Man, "A combined OFDM/SDMA approach," IEEE Journal on Selected Areas in Communications, vol. 18, no. 11, pp. 2312-2321, 2000

[3] L. Hanzo, Y. Akhtman, L. Wang, and M. Jiang, MIMO-OFDM for LTE, WIFI and WIMAX: coherent versus non-coherent and cooperative turbotransceivers. Wiley, 2010, (http://www-mobile.ecs.soton.ac.uk).

[4] J. Zhang, L. Hanzo, and X. Mu, "Joint decision-directed channel and noise-variance estimation for MIMO OFDM/SDMA systems based on expectation-conditional maximization," IEEE Transactions on Vehicular Technology, vol. 60, no. 5, pp. 2139-2151, 2011.

[5] S. Thoen, L. Deneire, L. Van der Perre, M. Engels, and H. De Man, "Constrained least squares detector for OFDM/SDMA-based wireless networks," IEEE Transactions on Wireless Communications, vol. 2, no. 1, pp. 129-140, 2003.

[6] X. Dai, "Pilot-aided OFDM/SDMA channel estimation with unknown timing offset," IEE Proceedings Communications, vol. 153, no. 3, pp. 392-398, 2006

[7] M. Jiang, J. Akhtman, and L. Hanzo, "Iterative joint channel estimation and multi-user detection for multiple-antenna aided OFDM systems," IEEE Transactions on Wireless Communications, vol. 6, no. 8, pp. 29042914, 2007.

[8] J. Ylioinas and M. Juntti, "Iterative joint detection, decoding, and channel estimation in turbo coded MIMO-OFDM," IEEE Transactions on Vehicular Technology, vol. 58, no. 4, pp. 1784-1796, 2009.

[9] J. Zhang, S. Chen, X. Mu, and L. Hanzo, "Joint channel estimation and multi-user detection for SDMA/OFDM based on dual repeated weighted boosting search," IEEE Transactions on Vehicular Technology, vol. 60, no. 7, pp. 3265-3275, 2011

[10] M. Dorigo and L. M. Gambardella, "Ant colony system: a cooperative learning approach to the traveling salesman problem," IEEE Transactions on Evolutionary Computation, vol. 1, no. 1, pp. 53-66, 1997.

[11] E. Zitzler and L. Thiele, "Multiobjective evolutionary algorithms: a comparative case study and the strength Pareto approach," IEEE Transactions on Evolutionary Computation, vol. 3, no. 4, pp. 257-271, 1999.

[12] J. H. Holland, "Genetic algorithms," Scientific American, vol. 267, no. 1, pp. 66-72, 1992.

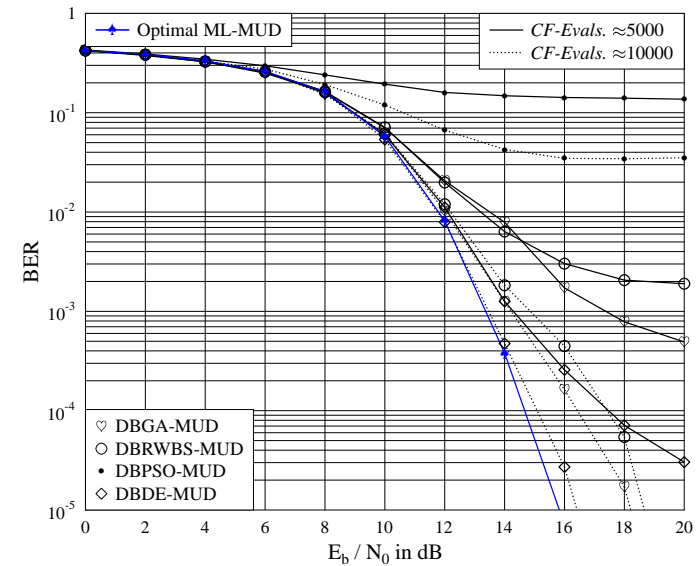

Fig. 7. BER performance of different discrete-valued binary stochastic optimization assisted MUDs using the parameters of Table I. The discretevalued binary stochastic optimization assisted MUDs optimize the objective function of Equation (8) by exploiting their intrinsic evolutionary mechanism. Hence the best achievable performance of them is the optimal ML-MUD, which is included as a lower bound benchmark.

[13] S. Chen, X. Wang, and C. J. Harris, "Experiments with repeating weighted boosting search for optimization in signal processing applications," IEEE Transactions on Systems, Man, And Cybernetics-Part B: CYBERNETICS, vol. 35, no. 4, pp. 682-693, 2005.

[14] J. Kennedy and R. Eberhart, "Particle swarm optimization," in IEEE International Conference on Neural Networks, 1995., vol. 4, 1995, pp. $1942-1948$.

[15] K. V. Price, R. M. Storn, and J. A. Lampinen, Differential evolution: a practical approach to global optimization. Springer Verlag, 2005.

[16] H. Ali, A. Doucet, and D. Amshah, "GSR: a new genetic algorithm for improving source and channel estimates," IEEE Transactions on Circuits and Systems I: Regular Papers, vol. 54, no. 5, pp. 1088-1098, 2007.

[17] K. Yen and L. Hanzo, "Genetic algorithm assisted joint multiuser symbol detection and fading channel estimation for synchronous CDMA systems," IEEE Journal on Selected Areas in Communications, vol. 19, no. 6 , pp. 985-998, 2001.

[18] "Genetic-algorithm-assisted multiuser detection in asynchronous CDMA communications," IEEE Transactions on Vehicular Technology, vol. 53, no. 5, pp. 1413-1422, 2004.

[19] C. Sacchi, M. Donelli, L. D'Orazio, R. Fedrizzi, and F. G. B. De Natale, "Genetic algorithm-based MMSE receiver for MC-CDMA systems transmitting over time-varying mobile channels," Electronics Letters, vol. 43, no. 3, pp. 172-173, 2007.

[20] K. K. Soo Y. M. Siu, W. S. Chan, L. Yang and R. S. Chen "Particleswarm-optimization-based multiuser detector for CDMA communications," IEEE Transactions on Vehicular Technology, vol. 56, no. 5, pp. 3006-3013, 2007.

[21] H. Liu and J. Li, "A particle swarm optimization-based multiuser detection for receive-diversity-aided STBC systems," IEEE Signal Processing Letters, vol. 15, pp. 29-32, 2008.

[22] B. Xu, "Particle-swarm-optimization based turbo multiuser detection for STBC MC-CDMA systems," in IEEE 19th International Symposium on Personal, Indoor and Mobile Radio Communications, 2008. PIMRC 2008., pp. $1-5$.

[23] M. Abuthinien, S. Chen, and L. Hanzo, "Semi-blind joint maximum likelihood channel estimation and data detection for MIMO systems," IEEE Signal Processing Letters, vol. 15, pp. 202-205, 2008.

[24] W. Yao, S. Chen, S. Tan, and L. Hanzo, "Minimum bit error rate multiuser transmission designs using particle swarm optimisation," IEEE Transactions on Wireless Communications, vol. 8, no. 10, pp. 50125017, 2009.

[25] W. Yao, S. Chen, and L. Hanzo, "Generalised MBER based vector precoding design for multiuser transmission," IEEE Transactions on Vehicular Technology, vol. 60, no. 2, pp. 739-745, 2011.

[26] R. C. Elliott and W. A. Krzymien, "Downlink scheduling via genetic algorithms for multiuser single-carrier and multicarrier MIMO systems with dirty paper coding," IEEE Transactions on Vehicular Technology, vol. 58, no. 7, pp. 3247-3262, 2009.

[27] L. M. O. Khanbary and D. P. Vidyarthi, "Reliability-based channel allocation using genetic algorithm in mobile computing," IEEE Transactions on Vehicular Technology, vol. 58, no. 8, pp. 4248-4256, 2009.

[28] L. Hanzo, M. Münster, B. J. Choi, and T. Keller, OFDM and MC-CDMA for broadband multi-user communications, WLANs, and broadcasting. Piscataway, NJ: IEEE Press, 2003, (http://www-mobile.ecs.soton.ac.uk).

[29] E. G. Larsson, "MIMO detection methods: how they work," IEEE Signal Processing Magazine, vol. 26, no. 3, pp. 91-95, 2009.

[30] X. Ma, H. Kobayashi, and S. C. Schwartz, "An EM-based channel estimation algorithm for space-time and space-frequency block coded OFDM," in 2003 IEEE International Conference on Acoustics, Speech, and Signal Processing, 2003. ICASSP'03., vol. 4, 2003, pp. 382-392. 